\title{
Neuroendocrinology 1967;2:192
}

\section{Book Review}

WILLHELM UMBACH : Elektrophysiologische und vegetative Phänomene bei stereotak-tischen Himoperationen. Springer, Berlin/Heidelberg/New York 1966. VI̋̄ +163 p., 54 fig., Price: DM 56 --.

In this monograph, Professor W. UMBACH, of the Clinic of Neurosurgery, University of Freiburg (Brsg), who has performed more than 2,600 stereotaxic operations himself, reviews work performed in this field during the last decade.

It is gratifying that, in addition to an abundance of neurophysiological findings, UMBACH presents new and interesting clinical data. He describes the use of steoreotaxic apparatus of RIECHERT and WOLF (1951) and RIECHERT an MUNDINGER (1956) (using a multi-contact electrode) for stimulation, coagulation and recording in extrapyramidal motor disease, pain, and psychological behavioral disturbances. In 35 patients with disorders of the extrapyramidal system, microelectrodes $(1.5-2 \mu)$ were used for unipolar recording from the nuclei v.o. thalami, in the globus pallidus and caudate nucleus. His discussion of the evaluation of EEG recordings during the different subcortical stimulations is mos interesting

The author's presentation of 500 successive stereotaxic stimulations and coagulations in the fornix, pallidum, and nuclei of the thalamus correlated with concomitant vegetative and psychic symptoms, is extremely instructive. The data refer particularly to intervention in diseases of the extrapyramidal system (Parkinson's syndrome, hyper- and dyskinesia) and chronic pain which is incurable by other methods; also mentioned are a few instances of intervention in erethic forms of schizophrenia and psychotic states with a tendency to self-destruction. The conclusion further a better understanding of psychosomatic abnormalities.

The second part of the book deals with observations made on 18 patients with temporal lobe epilepsy. Stereotaxic stimulation, coagulation, and EEG recordings, together with the observation of visceral, somatic, and affective alterations, are discussed in detail. The observations documented in this book are important to both clinical and experimental workers in neurology.

The book is written in German, but an English summary follows each chapter. 